\title{
TRAUMA NA GESTANTE: ANÁLISE DA MORTALIDADE MATERNA E FETAL
}

TRAUMA IN PREGNANCY: ANALYSIS OF MATERNAL AND FETAL MORTALITY

\author{
Paulo Roberto Corsi, TCBC-SP ${ }^{1}$ \\ Samir Rasslan, TCBC-SP ${ }^{2}$ \\ Liliana Bechelli de Oliveira ${ }^{3}$ \\ Flavia Souza Kronfly ${ }^{4}$ \\ Veruska Pereira Marinho ${ }^{4}$
}

\begin{abstract}
RESUMO: Foram analisadas retrospectivamente 26 pacientes gestantes traumatizadas, num período de nove anos. A média de idade foi 23,7 anos (16-42). A idade gestacional variou de dez a quarenta semanas (média 21,5 semanas), a maioria $(46,1 \%)$ no segundo trimestre. O mecanismo predominante $(65,3 \%)$ foi o trauma abdominal fechado por acidente automobilístico (atropelamento ou colisão). Na admissão, oito (30,7\%) pacientes apresentavam alterações hemodinâmicas. Seis doentes $(23,0 \%)$ apresentavam sangramento vaginal e, destas, quatro estavam hemodinamicamente normais. Analisamos a mortalidade materna, a mortalidade fetal e suas causas. Comparamos também a mediana dos valores do RTS e TRISS entre os grupos: sobrevida materno-fetal, sobrevida materna e óbito materno-fetal. Todas as gestantes admitidas com sangramento vaginal apresentaram óbito fetal. A mortalidade materna foi de 11,5\%, por choque hemorrágico. A mortalidade fetal foi de $30,7 \%$, sendo que $37,5 \%$ destes óbitos foram provocados pela morte materna. A principal causa de mortalidade fetal foi o descolamento de placenta $(50,0 \%)$. Os índices de trauma, RTS e TRISS, foram significativamente menor ( $p=0,0025$ e $p<0,0001$ ) no grupo óbito materno-fetal, porém esses índices não apresentaram valor prognóstico na mortalidade fetal.
\end{abstract}

Unitermos: Trauma; Gestação; Índices de Trauma; Mortalidade.

\section{INTRODUÇÃO}

Nas últimas seis décadas, observou-se uma grande modificação na incidência das causas de morte materna. No início do século, as principais causas eram as obstétricas, determinadas pela falta de cuidados pré-natais e inadequada assistência ao parto. Atualmente, com a melhoria dos serviços médicos, hospitalização dos partos e decréscimo da paridade, reduziu-se significativamente a mortalidade materna. O mesmo não foi observado em relação à taxa de mortalidade fetal, já que as causas não obstétricas passaram a assumir maior importância. ${ }^{1}$

Dentre as causas não obstétricas de mortalidade maternofetal, a mais freqüente é o trauma, sendo que $7 \%$ das mulheres grávidas sofrem algum tipo de traumatismo. ${ }^{1,2}$ Há uma tendência em aumentar este número em razão da maior exposição da mulher às situações de trauma. ${ }^{3}$
A incidência de traumas aumenta com a progressão da gravidez, até que no terceiro trimestre ocorrem traumatismos leves mais freqüentemente do que em qualquer outra época da vida adulta das mulheres. ${ }^{4}$

Os princípios e prioridades do atendimento são os mesmos de uma doente traumatizada não-gestante, embora as alterações fisiológicas da gestação modifiquem os sinais, os sintomas e o resultado dos exames laboratoriais. A gestação também pode alterar o padrão habitual de uma lesão traumática. ${ }^{5,6}$

A experiência do médico frente a essa ocorrência é pequena, uma vez que apenas $0,3 \%$ a $0,4 \%$ das gestantes necessitam hospitalização por trauma., ${ }^{7,8}$

Tendo em vista a pequena experiência individual e as casuísticas limitadas, este trabalho tem por objetivo discutir as particularidades do trauma na mulher grávida e avaliar as mortalidades materna e fetal decorrentes de traumatismos.

\footnotetext{
1. Professor Assistente.

2. Professor Titular. Diretor do Serviço de Emergência.

3. Acadêmica.

4. Médica Residente.
}

Recebido em 29/6/98

Aceito para publicação em 1/10/98

Trabalho realizado no Serviço de Emergência do Departamento de Cirurgia da Faculdade de Ciências Médicas da Santa Casa de São Paulo. 


\section{PACIENTES E MÉTODOS}

Foram analisados os prontuários de 26 gestantes traumatizadas atendidas de dezembro de 1988 a novembro de 1997 , no Serviço de Emergência do Departamento de Cirurgia da Faculdade de Ciências Médicas da Santa Casa de São Paulo. A média de idade foi de 23,7 anos (16-42). A idade gestacional variou de dez a quarenta semanas, sendo que nove casos $(34,6 \%)$ encontravam-se no primeiro trimestre, $12(46,1 \%)$ no segundo e cinco $(19,2 \%)$ no terceiro trimestre de gestação.

O trauma fechado ocorreu em $21(80,7 \%)$ gestantes, sendo dez $(38,4 \%)$ atropelamentos, sete $(26,9 \%)$ colisões de veículos e quatro $(15,3 \%)$ quedas acidentais. Três $(11,5 \%)$ foram vítimas de ferimento por arma branca e duas $(7,6 \%)$ de ferimento por projétil de arma de fogo (Tabela 1).

Tabela 1

Mecanismos de trauma

\begin{tabular}{c|c}
\multicolumn{2}{c}{ Mecanismos de trauma } \\
\hline Tipo de trauma & $\begin{array}{l}\text { Gestantes } \\
\text { Número }(\%)\end{array}$ \\
\hline Trauma fechado & $21(80,7)$ \\
Atropelamento & $10(38,4)$ \\
Colisão & $7(26,9)$ \\
Quedas & $4(15,3)$ \\
Espancamento & -- \\
Outros & $5(19,2)$ \\
Trauma aberto & $2(7,6)$ \\
Arma de fogo & $3(11,5)$ \\
\hline Arma branca & $26(100)$ \\
\hline
\end{tabular}

Na admissão, oito $(30,7 \%)$ pacientes apresentavam alterações hemodinâmicas: em quatro gestantes, a freqüência cardíaca era superior a 100 batimentos por minuto, numa gestante a pressão sistólica era inferior a $100 \mathrm{mmHg}$ e, em três, havia hipotensão e taquicardia.

Seis doentes $(23,0 \%)$ apresentavam sangramento vaginal e, destas, quatro estavam hemodinamicamente normais.

Foram realizadas duas cesáreas. Numa gestante com lesão de baço e choque hemorrágico, ocorreu óbito fetal, e a cesárea teve intenções táticas, para facilitar a exploração da cavidade (caso 22). A segunda, paciente com lesão hepática, após a cesárea, o feto a termo foi encaminhado ao berçário em bom estado (caso 23). Utilizamos em todas as gestantes os índices de trauma: Revised Trauma Score (RTS), ${ }^{9}$ o Injury Severity Score (ISS) ${ }^{10}$ e o Trauma Revised Injury Severity Score (TRISS). ${ }^{11}$ Analisamos a mortalidade materna, a mortalidade fetal e suas causas. Comparamos também a mediana dos valores do RTS e TRISS entre os grupos: sobrevida maternofetal, sobrevida materna e óbito materno-fetal.

\section{RESULTADOS}

A mortalidade materna foi de $11,5 \%$ (três casos), provocada por choque hemorrágico. A mortalidade fetal foi de 30,7\%
Tabela 2

Relação entre os tipos de trauma e a mortalidade materno-fetal

\begin{tabular}{lcccc}
\hline Tipo de trauma & Número (\%) & Óbitos maternos & Óbitos fetais \\
\hline Trauma fechado & $21(80,6)$ & 2 & 7 \\
Atropelamento & $10(38,4)$ & 2 & 6 \\
Colisão de veículo & $7(26,9)$ & - & 1 \\
Quedas & $4(15,3)$ & - & - \\
Trauma aberto & $5(19,1)$ & 1 & 1 \\
Arma de fogo & $2(7,6)$ & 1 & 1 \\
Arma branca & $3(11,5)$ & - & - \\
\hline Total & $26(100)$ & $3(11,5 \%)$ & $8(30,7 \%)$ \\
\hline
\end{tabular}

(oito casos), sendo que 37,5\% destes acompanhados de mortalidade materna (Tabela 2).

Treze $(50 \%)$ das gestantes traumatizadas foram operadas. Doze foram submetidas a laparotomia exploradora e uma a toracotomia exploradora. Hematoma retroperitoneal foi encontrado em nove das $12(75,0 \%)$ doentes submetidas a laparotomia, além de outras lesões representadas na tabela 3. As três pacientes que faleceram foram submetidas a cirurgia de urgência. As 13 gestantes não operadas eram portadoras de traumas de extremidades, ferimentos cortantes, escoriações e outras lesões menores, e sua evolução ocorreu sem óbito fetal.

Tabela 3

Tipo de trauma, achado cirúrgico e mortalidade materno-fetal

\begin{tabular}{|c|c|c|c|c|}
\hline Gestante & $\begin{array}{l}\text { Tipo de } \\
\text { trauma }\end{array}$ & Achado Cirúrgico & $\begin{array}{l}\text { Óbito } \\
\text { Materno }\end{array}$ & $\begin{array}{l}\text { Óbito } \\
\text { Fetal }\end{array}$ \\
\hline 1 & A & $\begin{array}{l}\text { hematoma retroperitoneal, } \\
\text { lesão de cólon }\end{array}$ & + & + \\
\hline 3 & $\mathrm{C}$ & hematoma retroperitoneal & - & + \\
\hline 6 & FAB & $\begin{array}{l}\text { hematoma retroperitoneal } \\
\text { lesão intestinal }\end{array}$ & - & - \\
\hline 8 & FAF & $\begin{array}{l}\text { hematoma retroperitoneal, } \\
\text { lesão de intestino, útero, } \\
\text { bexiga, ureter, vasos ilíacos }\end{array}$ & + & + \\
\hline 9 & FAF & lesão pulmonar & - & - \\
\hline 10 & A & $\begin{array}{l}\text { hematoma retroperitoneal, } \\
\text { lesão de baço, fratura de bacia }\end{array}$ & 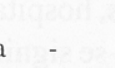 & + \\
\hline 11 & A & $\begin{array}{l}\text { hematoma retroperitoneal, } \\
\text { fratura de bacia }\end{array}$ & + & + \\
\hline 16 & FAB & $\begin{array}{l}\text { hematoma retroperitoneal, } \\
\text { lesão intestinal }\end{array}$ & - & - \\
\hline 20 & A & $\begin{array}{l}\text { hematoma retroperitoneal, } \\
\text { hematoma de } \\
\text { anexos esquerdos }\end{array}$ & - & + \\
\hline 21 & A & $\begin{array}{l}\text { hematoma retroperitoneal, } \\
\text { lesão hepática e lesão renal }\end{array}$ & - & + \\
\hline 22 & A & $\begin{array}{l}\text { lesão de baço, hematoma peri- } \\
\text { esplênico (cesárea) }\end{array}$ & - & + \\
\hline 23 & FAB & lesão hepática (cesárea) & - & - \\
\hline 26 & $\mathrm{C}$ & hérnia diafragmática & - & - \\
\hline
\end{tabular}

A: Aberto; C: Contuso; FAB: Ferimento por arma branca; FAF: Ferimento por arma de fogo

Todas as seis $(23,0 \%)$ gestantes admitidas com sangramento vaginal apresentaram óbito fetal. Porém, tal sangramento não esteve presente em $25,0 \%$ dos óbitos fetais. A mortalidade fetal ocorreu em três casos por morte materna. 
O descolamento de placenta foi a causa da morte de quatro fetos cujas mães apresentaram sangramento vaginal à admissão sem alteração hemodinâmica e TRISS superior a 0,97. No outro caso, choque hemorrágico prolongado materno determinou o óbito fetal (Tabela 4).

Tabela 4

Causas de morte fetal

\begin{tabular}{lc}
\hline Causas de morte & Número (\%) \\
\hline Descolamento de placenta & $4(50,0)$ \\
Morte materna & $3(37,5)$ \\
Choque prolongado & $1(12,5)$ \\
\hline Total & $8(100)$ \\
\hline
\end{tabular}

A análise comparativa entre RTS e TRISS nos grupos: sobrevida materno-fetal, sobrevida materna e óbito materno fetal, por meio do teste de Kruskall-Wallis, mostrou diferença estatisticamente significativa entre os grupos sobrevida materno-fetal e óbito materno-fetal. O teste não-paramétrico de Dunn mostrou que não houve diferença entre os grupos sobrevida materno-fetal e sobrevida materna (Tabela 5).

Tabela 5

Valores de RTS e TRISS nos diferentes grupos, expressos em mediana.

\begin{tabular}{lcccc}
\hline \multicolumn{1}{c}{ Grupos } & Número de casos & RTS & TRISS \\
\hline Sobrevida materna e fetal & 18 & $7,84^{*}$ & $1,00^{*}$ \\
Sobrevida materna & 5 & $7,55^{*}$ & $0,98^{*}$ \\
Óbito materno e fetal & 3 & 6,81 & 0,88 \\
\hline$p$ (Kruskall-Wallis) & 0,0025 & $<0,0001$ \\
$*$ não houve diferença significativa (teste não-paramétrico de Dunn).
\end{tabular}

\section{DISCUSSÃO}

O trauma é a principal causa não-obstétrica de mortalidade materna em gestantes assim como nas mulheres nãográvidas em idade reprodutiva., ${ }^{1,2,4,5}$

O trauma abdominal fechado é o tipo de trauma mais freqüentemente encontrado. A maioria dos autores demonstrou que o agente mais comum é o acidente automobilístico, seguido de ferimentos por projétil de arma de fogo, ferimentos por arma branca e suicídios. ${ }^{2,3,12-16}$

Na nossa casuística, os acidentes automobilísticos foram a principal causa de trauma em gestantes, seguida das quedas, ocorrência muito freqüiente durante a gestação. Helton et $\mathrm{al}^{29}$ relataram que uma em cada 12 mulheres sofrem queda durante a gestação. Segundo Baker, ${ }^{3}$ a maioria destes traumas é leve e não requer hospitalização.

Não foi observado nenhum caso de espancamento, embora a agressão tenha sido relatada como um dos mecanismos de trauma em gestantes. Quando presente, a agressão, na maioria das vezes, é realizada pelo marido ou namorado. . $2,30^{2}$

Não temos informatizados todos os atendimentos desse período, porém, comparamos o tipo de trauma nas 26 gestantes atendidas num período de nove anos, com 205 pacientes não-gestantes, de mesma faixa etária atendidas num período de um ano. Não houve diferença estatística entre os grupos, mostrando que a gestante está sujeita aos mesmos tipos de trauma que a não-gestante.

O efeito do trauma na gestação depende da idade gestacional, intensidade de agressão materno-fetal, tipo e gravidade do trauma. ${ }^{17}$ Está associado a uma alta taxa de perda fetal, sendo que as causas mais comuns são o descolamento da placenta e a mortalidade materna. Portanto, a perda fetal pode ocorrer mesmo que o feto não tenha sido diretamente atingido. ${ }^{13}$ A perda volêmica pelo trauma provoca uma redução do fluxo sangüíneo preferencialmente na placenta, pela sua sensibilidade às catecolaminas. Pode haver grave sofrimento fetal sem sinais clínicos de choque na mãe, cuja volemia sofre significativo aumento no final da gestação. ${ }^{3,5,6,18,19}$ Por estes motivos é que o melhor tratamento para o feto é o restabelecimento das funções vitais da mãe. ${ }^{20} \mathrm{O}$ traumatismo fetal sem comprometimento materno é raro. ${ }^{21}$

A mobilidade e elasticidade do útero protege-o quando ocorre contusão abdominal. Devido ao líquido amniótico, a força se distribui em todas as direções, mas a rotura pode ocorrer no fundo posterior, parte de maior fragilidade. No final da gravidez, quando a cabeça está encaixada na pelve e o líquido amniótico está diminuído, geralmente o trauma abdominal oferece maior gravidade. ${ }^{6}$

A rotura uterina é uma complicação infreqüente, porém, com mortalidade próxima dos $100 \% .{ }^{22}$ Elias $^{23}$ descreveu um caso de rotura uterina por acidente automobilístico, no qual a mãe foi lançada fora do veículo através do pára-brisa, sofrendo grave laceração da parede abdominal e do útero. O bebê foi encontrado com vida fora do útero materno (cesárea traumática).

Quando a mãe sobrevive, os danos placentários assumem maior importância na mortalidade fetal..$^{2,8,20}$ Neste caso, encontra-se com maior freqüência o descolamento de placenta, que ocorre dentro das 48 horas após o trauma, mas pode ocorrer até cinco dias mais tarde. ${ }^{24} \mathrm{~A}$ diferença entre a elasticidade da placenta e do útero pode provocar descolamento por desaceleração. Os achados clínicos comuns neste evento incluem dor abdominal, perda de líquido amniótico, contração uterina, sangramento vaginal, acidose e, em relação ao feto,

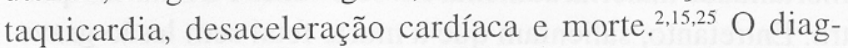
nóstico imediato de dano placentário é da maior importância, pois a intervenção precoce pode alterar o prognóstico do feto. ${ }^{2}$

O fornecimento de oxigênio e a reposição volêmica devem ser realizados mesmo nas doentes hemodinamicamente normais com trauma leve. ${ }^{19} \mathrm{O}$ útero gravídico produz obstrução mecânica significativa ao retorno venoso e, embora a reanimação seja mais bem realizada na posição supina, essa posição produz obstrução da veia cava superior com um declínio de $25 \%$ do débito cardíaco.

Apesar da mortalidade materna ser elevada, esse índice não é superior ao das mulheres não-grávidas traumatizadas. ${ }^{16,19,25,26}$ Entretanto, a mortalidade fetal é superior à 
mortalidade materna numa proporção que varia de $3: 1$ a $9: 1 .^{16,20,26-28}$

A extensão e a gravidade da lesão materna e fetal nos casos de ferimento por projétil de arma de fogo dependem de vários fatores, como as características do projétil, distância, ângulo de entrada, trajetória e idade gestacional que altera a posição das vísceras abdominais. ${ }^{22} \mathrm{~A}$ baixa velocidade de penetração do projétil num abdome de gestação avançada geralmente transfere uma maior energia para o útero e seu conteúdo. A morte fetal é resultado comum nos ferimentos por arma de fogo, enquanto a materna é rara, pois o útero protege os outros órgãos abdominais. ${ }^{6,13}$

No nosso estudo, a presença de sangramento vaginal esteve correlacionada com morte fetal em $100 \%$ dos casos.

Lesões diretas no feto são complicações comuns do trauma durante a gestação. Ocorrem mais freqüentemente no final da gestação, período em que o pólo cefálico está encaixado na pelve materna e que, por esse motivo, freqüentemente resulta em fratura craniana ou hematoma cerebral, principalmente quando há fratura de pelve materna, ou quando há compressão do feto entre o promontório sacral da mãe e o cinto de segurança do veículo. Essa lesão é causada pela interação da força dę desaceleração quando a mãe flexiona seu corpo para a frente. ${ }^{16,25}$

É freqüiente encontrar na gestação a ocorrência de lesões esplênicas e sérias hemorragias retroperitoneais na parte baixa do abdome e pelve como resultado do aumento do tamanho uterino, do fluxo sangüíneo regional e dilatação dos vasos pélvicos. ${ }^{6}$ Rothenberger et al, ${ }^{16}$ analisando 103 casos de trauma na gestação, encontraram choque hemorrágico em todos os casos de morte materna e este foi responsável por $80 \%$ da mortalidade fetal. Na nossa casuística, a mortalidade materna foi devida a choque hemorrágico, e a maioria das pacientes operadas apresentava hematoma retroperitoneal.

Tentando diminuir a incidência de complicações decorrentes do trauma abdominal fechado, foi estudada por vários autores a eficácia do cinto de segurança. ${ }^{19,25}$ Crosby \& Costiloe, ${ }^{25}$ em 1971, verificaram que o cinto diminui a mortalidade materna e fetal, já que impede a ejeção da mulher para fora do veículo. Acrescentaram que, quando isto ocorre, a mortalidade materna aumenta em sete vezes e a fetal em quatro. Entretanto, salientam que a morte fetal sem lesão grave na mãe pode ocorrer devido ao uso do cinto. Crosby et $a l,{ }^{31}$ em 1968, em estudo experimental com babuínos, mostraram que o uso do cinto de três pontos pode reduzir significativa- mente a mortalidade fetal, prevenindo os efeitos de compressão, dissipando as forças de desaceleração para uma área maior e evitando a flexão do corpo materno sobre a bacia. Desta forma, a taxa de perda fetal, que com o cinto de dois pontos é aproximadamente $50 \%$, é reduzida para cerca de $8 \%$.

A cesárea está indicada quando o feto for viável e as condições maternas desfavoráveis. Apenas deve ser interrompida a gravidez com feto inviável quando o útero interferir no tratamento das lesões maternas. Nas lesões uterinas com feto viável, também deve ser feita a cesárea, pela freqüente ocorrência de trabalho de parto prematuro. O trauma sobre o útero provoca a contração uterina e a rotura da bolsa. Se o feto for inviável, deve-se reparar o útero, com a intenção de progredir a gravidez. ${ }^{5}$

A literatura mostra que a análise do prognóstico maternofetal pode ser feita a partir de índices de trauma. Observouse que a mortalidade fetal está relacionada com altos valores no ISS, AIS, acidose e hipoxia maternas. ${ }^{27,28}$ Existe também, relação entre a mortalidade fetal e a presença de CIVD materna. ${ }^{8}$ Por outro lado, não é infreqüente ocorrer morte fetal com ISS igual a zero. ${ }^{30}$

No Serviço de Emergência utilizamos o RTS, o ISS e o TRISS, índices rotineiramente adotados para os doentes traumatizados. Esses índices avaliaram corretamente o prognóstico materno, porém, assim como outros autores, ${ }^{28,32}$ observamos que o RTS e o TRISS não apresentaram valor prognóstico na mortalidade fetal. Um trauma abdominal com lesão exclusiva uterina, sem risco materno, pode ser grave ou mesmo fatal para o feto. Da mesma forma, traumas de menor intensidade sem alterações sistêmicas graves para a mãe podem ter influências significativas no prognóstico do feto.

A análise desta pequena casuística de gestantes traumatizadas permite as seguintes considerações:

a) A mortalidade fetal foi maior que a mortalidade materna.

b) A principal causa de mortalidade fetal foi o descolamento de placenta e a mortalidade materna ocorreu por choque hemorrágico.

c) Chama a atenção que todas que apresentavam sangramento vaginal tiveram óbito fetal. Isto significa que elas tinham descolamento de placenta na ocasião da admissão.

d) A utilização dos índices de gravidade (RTS e TRISS) não mostrou valor prognóstico em relação à mortalidade fetal. Isto porque, uma gestante sem repercussões graves pode evoluir com óbito fetal.

\footnotetext{
ABSTRACT

Twenty-six traumatized pregnant patients were retrospectively analyzed over a nine year period. The average age was 23.7 years (16-42). Gestational age was 10-40 weeks (average 21.5 weeks), the majority (46.1\%) being in the second trimester. The predominant mechanism (65.3\%) was blunt abdominal trauma by automobile accident (collision or running over). During admission, eight (30.7\%) patients showed hemodynamic alterations. Six patients (23.0\%) showed vaginal bleeding
} 
and of these, four were hemodynamically normal. Median values of RTS and TRISS were also compared among the following groups: maternal-fetal survival, maternal survival and maternal-fetal death. All pregnant women admitted with vaginal bleeding showed fetal death. Maternal mortality was caused by hemorrhagic shock in $11.5 \%$ of the cases. Fetal mortality occurred in $30.7 \%$ of the cases, and $37.5 \%$ of these were caused by maternal death. The main cause of the fetal death (50.0\%) was abruptio placentae. RTS and TRISS were significantly lower $(p=0.0025$ and $p<0.0001)$ in the maternal-fetal death group, but these scores presented no prognostic value for fetal mortality.

Key Words: Trauma; Pregnancy; Trauma Score; Mortality.

\section{REFERÊNCIAS}

1) Peckham C, King R - A study of intercurrent conditions observed during pregnancy. Am J Obstet Gynecol 1963;87:609-24.

2) Pearlman MD - Motor vehicle crashes, pregnancy loss and preterm labor. Int J Gynecol Obstet 1997;57:127-32.

3) Baker DP - Traumatismo na paciente grávida. Clin Cir Am Norte 1982; 62:313-29.

4) McAnena OJ, Moore EE, Marx JA - Avaliação inicial do paciente com traumatismo abdominal contuso. Clin Cir Am Norte 1990;70: 509-31.

5) American College of Surgeons: "Trauma in Pregnancy". In Advanced Trauma Life Support Instructor Manual. USA 1993 - Chap.11: 283-92.

6) Moise Jr KJ, Belfort MA - Emergência controlada para a paciente obstétrica. Clin Cir Am Norte 1997;77:839-45.

7) Lavin Jr JP, Polsky SS - Abdominal trauma during pregnancy. Clin Perinatol 1983;10:423-38.

8) Ali J, Yeo A, Gana TJ, et al - Predictors of fetal mortality in pregnant trauma patients. J Trauma 1997;42:782-5.

9) Champion HR, Sacco WJ, Copes WS, et al - A revision of the Trauma Score. J Trauma 1989;29:623-9.

10) Copes WS, Champion HR, Sacco WJ et al - The Injury Severity Score revisited. J Trauma 1988;28:69-75.

11) Boyd CR, Tolson MA, Copes WS - Evaluation trauma care: The TRISS Method. J Trauma 1987;27:370-8.

12) Connolly AM, Katz VL, McMahon MJ et al - Trauma and pregnancy. Am J Perinatol 1997;14:331-6.

13) Morkovin V - "Trauma in pregnancy". In Farrel,R.G. (ed.): $O B / G Y N$ Emergencies: The first 60 minutes. Rockville - USA - Aspen Publications, 1986,6:pp.71-86.

14) Goodwin TM, Breen MT - Pregnancy outcome and fetomaternal hemorrhage after noncatastrophic trauma. Am J Obstet Gynecol 1990;162:665-71.

15) Pearlman MD, Tintinalli JE, Lorenz RP - A prospective controlled study of outcome after trauma during pregnancy. Am J Obstet Gynecol 1990;162:1.502-10.

16) Rothenberger D, Quattlebaum FW, Perry Jr JF, et al - Blunt maternal trauma: a review of 103 cases. J Trauma 1978;18:173-9.

17) Pearlman MD, Tintinalli JE, Lorenz RP - Blunt trauma during pregnancy. N Engl J Med 1990;323:1.609-13.

18) Lukaski HC, Siders WA, Nielsen EJ, et al - Total body water in pregnancy: assessment by using bioelectrical impedance. Am J Clin Nutr 1994:59:578-85.
19) Esposito TJ, Gens DR, Smith LG, et al - Trauma during pregnancy: A review of 79 cases. Arch Surg 1991;126:1.073-8.

20) Kissinger DP, Rozycki GS, Morris Jr JA, et al - Trauma in pregnancy: predicting pregnancy outcome. Arch Surg 1991;126:1.079-86.

21) Maciel CJ, Leite MSB - Traumatismo fetal intra-útero sem comprometimento materno (relato de um caso) Rev Goiana Med 1983; 29:179-81

22) A.C.O.G. - Technical bulletin number 161: Trauma during pregnancy. Int J Gynecol Obstet 1993;40:165-70.

23) Elias M - Rupture of the uterus by external violence. Lancet 1950;2: 253-4.

24) Lifschultz BD, Donoghue ER - Fetal death following maternal trauma: Two case reports and survey of the literature. J For Sci 1991; 36: $1.740-4$.

25) Crosby WM, Costiloe JP - Safety of lap-belt restraint for pregnant victims of automobile collisions. N Engl J Med 1971;284:632-6.

26) Timberlake GA, McSSwain NE - Trauma in pregnancy: a 10-year perspective. Am Surg 1978;55:151-3

27) Drost TF, Rosemurgy AS, Sherman HF, et al - Major trauma in pregnant women: maternal/fetal outcome. J Trauma 1990;30:574-8.

28) Hoff WS, D'Amelio LF, Tinkoff GH, et al - Maternal predictors of fetal demise in trauma during pregnancy. Surg Gynecol Obstet 1991; 172:175-80.

29) Helton AS, McFarlane J, Anderson ET - Battered and pregnant: A prevalence study. Am.J.Public Health 1987;77:1.337-9.

30) Poole GV, Martin Jr JN, Perry Jr KG, et al - Trauma in pregnancy: the role of interpersonal violence. Am J Obstet Gynecol 1996;174: 1.873-7.

31) Crosby WM, Snyder RG, Snow CC, et al - Impact injuries in pregnancy. Experimental studies. Am J Obstet Gynecol 1968;101:100-10.

32) Biester EM, Tomich PG, Esposito TJ, et al - Trauma in pregnancy: Normal Revised Trauma Score in relation to other markers of maternofetal status - A preliminary study. Am J Obstet Gynecol 1997; 176:1.206-12.

\section{ENDEREÇO PARA CORRESPONDÊNCIA}

Dr. Paulo Roberto Corsi

Rua Joaquim Antunes, 577/132

05415-011 - São Paulo-SP 